\title{
CALCULO DE PESSOAL DE ENFERMAGEM: COMPETENCIA DA ENFERMEIRA
}

\author{
Maria Coeli Campedelli * \\ Clarinda Takito** \\ Tania Regina Sancinetti*** \\ Maria Antonieta Benko ****
}

\begin{abstract}
CAMPEDELLI, M.C.; TAKITO, C.; SANCINETTI, T.R.; BENKO, M.A. Cálculo de pessoal de enfermagem: competência da enfermeira. Rev. Esc. Enf. USP, São Paulo, $21(1): 3-15$, abr. 1987.
\end{abstract}

As autoras analisam a problema enfrentado pela Direção de Enfermagem de um hospital, referente ao cálculo do número de pessoal necessário para a prestação da assistência de enfermagem aos pacientes. Referem que os hospitais, numa fase inicial, poderão utilizar-se de fórmulas encontradas na literatura, mas enfatizam que, posteriormente, os cálculos devem ser refeitos, levando em consideração o número de horas diárias de cuidados que, naquela situação, os pacientes requerem, $e$ outros fatores locais que interferem na assistência de enfermagem.

UNITERMOS: Pessoal de enfermagem em hospitais. Administração em enfermagem. Serviços de enfermagem em hospitais.

\section{INTRODUÇAOO}

No desempenho de seu papel profissional, a enfermeira tem buscado continuamente assistir à saúde do homem de forma cada vez mais cientifica e independente. Esta busca ocorre tanto em situações novas, como no reestudo daquelas que a prática tem como consagradas. A dotação de recursos humanos para a prestação da assistência de enfermagem é uma das suas atribuições já estabelecidas, mas que constitui, muitas vezes, um problema para a enfermeira que responde pela gerência da assistência nos hospitais onde se discute o assunto. Inúmeras são as dificuldades encontradas para o desempenho desta coordenação.

$O$ pessoal de enfermagem representa um percentual importan. te em relação ao número total dos servidores no hospital. Segundo FELDMANN ${ }^{5}$ e DUTRA ${ }^{4}$, este índice é igual a $60 \%$, o que representa um custo elevado e envolve um processo contínuo de admissão, seleção,

* Enfermeira. Professor Assistente Doutor da Disciplina Fundamentos de Enfermagem da Escola de Enfermagem da USP.

** Enfermeira. Professor Assistente da Disciplina Fundamentos de Enfermagem da Escola de Enfermagem da USP e Diretora do Departamento de Assistencia de Enfermagem do Hospital Universitário da USP.

*** Enfermeira. Diretora de Serviço do Pronto Atendimento do Departamento de Assistencia de Enfermagem do Hospital Universitário da USP.

**** Enfermeira. Diretor Técnico de Divisăo do Departamento de Assistência de Enfermagem do Hospital Universitário da USP. 
treinamento, aperfeiçoamento e avaliação. Provoca, por motivos econômicos, políticos e outros, a interferência de profissionais de outras áreas na determinação da quantidade e qualidade do pessoal. Outra dificuldade que pode ocorrer é a ausência ou indefinição de programas e objetivos da Instituição ou do conflito desta com os objetivos do serviço de enfermagem. $E$ necessário identificar a causa da interferência que, não raro, leva à ingerência, para demonstrar os riscos operacionais de um serviço programado inadequadamente e o comprometimento legal que a Instituição assume diante dos inúmeros perigos e falhas, que ocorrem tanto com o paciente quanto com o pessoal que o assiste. A dotação de pessoal, portanto, além de ser da competência da enfermeira, representa uma preocupação constante para a enfermagem, visto que está diretamente ligada ao produto final de seu trabalho - a qualidade da assistência prestada e a segurança do paciente.

A preocupação com a qualidade da assistência de enfermagem a ser prestada é enfatizada por diversos autores, entre os quais ALCALA ${ }^{2}$. DUTRA ${ }^{4}$, OGUISSO ${ }^{7}$ e FELDMANN. ${ }^{5}$. Este assunto requer da enfermeira conhecimento específico, habilidade na sua condução e segurança na justificativa de suas propostas. Estas propostas são encontradas em estudos realizados e na análise de situações vivenciadas.

O tempo necessário à prestação da assistência de enfermagem varia segundo autor e tipo de paciente. De acordo com OGUISSO 7, a necessidade de hora/enfermagem é a seguinte nas diferentes clínicas: Pediatria 4,9 h; Clínica Médica 2,2 h; Clínica Cirúrgica 2,4 h; U. T. I. 7,1 h. FELDMANN ${ }^{5}$ estabelece, para a Pediatria, 4,9 h; Clínica Médica 3,4 h; Clínica Cirúrgica $3,5 \mathrm{~h}$; Obstetrícia $4,2 \mathrm{~h}$. ABBOUD ${ }^{1}$ adota os mesmos indices de FELDMANN ${ }^{5}$, mas acrescenta $12 \mathrm{~h}$ para a U.T.I.; ATALA ${ }^{3}$ também apresenta valores aproximados: Pediatria 3 a $4 \mathrm{~h}$; Clinica Médica 2,5 a 3h; Clínica Cirúrgica $3 \mathrm{~h}$; Obstetrícia $2 \mathrm{~h}$ e U.T. I. 12,5 h. Já em 1982, ALCALA ${ }^{2}$ realizou um estudo para determinar o quadro de pessoal da Superintendência Médico Hospitalar de Urgência do Município de S. Paulo, partindo do tipo de cuidado prestado a cada paciente, com base na filosofia do Cuidado Progressivo ao Paciente. Definiu a hora/enfermagem para o paciente Adulto e Pediátrico: paciente adulto, cuidados minimos $2,5 \mathrm{~h}$; intermediário $4,5 \mathrm{~h}$; semi-intensivo $10 \mathrm{~h}$ e intensivo $18 \mathrm{~h}$ e para a Pediatria $4,5 \mathrm{~h} ; 5,5 \mathrm{~h} ; 10 \mathrm{~h}$ e $18 \mathrm{~h}$ respectivamente. Pode-se observar que a variação da hora/enfermagem entre os diversos autores é pequena, e muitas vezes não traduz a realidade do dia-a-dia, que pode oscilar muito de acordo com a gravidade dos pacientes, exceção feita a ALCALA $^{2}$ que se preocupou exatamente com este aspecto.

Com relação ao percentual de distribuição por categoria funcional há também diversidade de opinião entre os diversos autores: ALCALA 2 preconiza $15 \%$ de enfermeiros, $45 \%$ de auxiliares de enfermagem e $40 \%$ de atendentes; FELDMANN ${ }^{5}$ recomenda $10 \%, 20 \%$ e $60 \%$ respectivamente. No material preparado pelo professor Miguel ABBOUD ${ }^{1}$ para o Curso de Administração Hospitalar da Escola de Administração de Empresas de São Paulo, da Fundação Getúlio Vargas, e do Hospital das Clí- 
nicas da Faculdade de Medicina da Universidade de São Paulo, o preconizado para todas as unidades, incluindo emergência, é $5 \%$ de enfermeiros, $10 \%$ de técnicos de enfermagem, $25 \%$ de auxiliares de enfermagem e $60 \%$ de atendentes; para as Unidades de Terapia Intensiva, este percentual tem a seguinte distribuição: $10 \%$ de enfermeiros, $20 \%$ de técnicos de enfermagem, $60 \%$ de auxiliares de enfermagem e $10 \%$ de atendentes.

Quando este percentual é discutido a partir do ponto de vista administrativo de custo, deve ser levada em conta a qualidade da assistência que se pretende prestar, pois esta é diretamente dependente da qualificação profissional e ocupacional dos funcionários.

O Hospital Universitário da Universidade de São Paulo (HU) conta atualmente com 1252 cargos de funcionários, dos quais 450 são destinados à enfermagem, o que representa $35,94 \%$ do pessoal, portanto um percentual abaixo do preconizado. A distribuição funcional é a seguinte: 100 enfermeiros $(22,22 \%), 15$ técnicos de enfermagem $(3,33 \%), 101$ auxiliares de enfermagem $(22,44 \%), 213$ atendentes de enfermagem $(47,33 \%), 19$ escriturários $(4,22 \%)$ e 01 recreacionista $(0,2 \%)$.

Se forem retiradas do quadro do Hospital Universitário as duas categorias não tradicionalmente incluídas no quadro funcional de enfermagem (19 escriturários e 01 recreacionista), teremos 430 funcionários distribuídos da forma seguinte: $23 \%$ de enfermeiros, $3,5 \%$ de técnicos de enfermagem, $23,5 \%$ de auxiliares de enfermagem e $50 \%$ de atendentes de enfermagem.

O Departamento de Assistência de Enfermagem do Hospital Uni. versitário tem estabelecido, em sua filosofia, a adoção de um sistema de assistência de enfermagem para o desempenho das atividades junto ao paciente, o qual, com a prescrição diária do cuidado, está vinculado ao cumprimento de um dispositivo legal do exercício profissional da enfermeira, segundo a qualidade da assistência a ser prestada e deve ser considerado na elaboração do quadro de pessoal.

A medida que se procura diferenciar o cuidado de enfermagem necessário a cada paciente, varia o número de horas de enfermagem gasto e, como conseqüência, a quantidade e à qualidade de pessoal também será diferente. Esta variação é questionada e por isso é necessário sejam estipulados padrōes que, em consonância com a filosofia do serviço, mobilizem o tipo de assistência que se pretende prestar. A vivência desta problemática deu origem ao presente trabalho, que tem como objetivo maior a estimativa do número de funcionários de enfermagem necessário à prestação da assistência de enfermagem no Hospital Universitário da Universidade de São Paulo, sendo, para isso, utilizados três índices diferentes. A fim de se realizar este objetivo, foram adotados, para cálculo, dois indices propostos por diferentes autores: 1 . ABBOUD ${ }^{1}$ 2. ALCALA 2 e 3 terceiro índice utilizado foi extraído da realidade identificada no Hospital Universitário. 


\section{METODOLOGIA}

O estudo foi realizado no Hospital Universitário da Universidade de São Paulo em duas clínicas de pacientes internados: Clínica Médica e Clínica Pediátrica, localizadas respectivamente no $5^{\circ}$ e $4^{\circ}$ pavimentos.

A população de estudo foi constituida por todos os pacientes hospitalizados nas duas clínicas, durante o período de um mês (maio de 1986).

As duas clínicas que constituíram o campo de estudo têm gerência própria de pessoal, material e movimentação de pacientes.

Cada uma delas conta com duas unidades de internação, posto de enfermagem, sala de serviço e outras dependências próprias de uma unidade de internação; o número de leitos em ambas as clínicas, no mês de estudo, foi de 35 .

Os leitos, na Clínica Médica, estão distribuídos em quartos individuais e enfermarias de 5 leitos, estando uma delas equipada para cuidados especiais a pacientes graves.

A Clínica Pediátrica é constituída também por duas unidades de internação com enfermarias de 5 leitos, quartos individuais, uma enfermaria para 12 berços e uma unidade para cuidados especiais.

Os quartos individuais, nas duas clínicas, são geralmente ocupados por pacientes que requerem algum tipo de isolamento fisiológico ou emocional.

Para cálculo do número de pessoal necessário foi utilizada sempre a mesma fórmula:

\begin{tabular}{|c|c|c|c|c|c|}
\hline $\begin{array}{l}\text { «leitos } \\
\text { ocupados» }\end{array}$ & $x$ & $\begin{array}{l}\text { «horas de assistência } \\
\text { de enfermagem» }\end{array}$ & $x$ & $\begin{array}{l}\text { «dias da } \\
\text { semana» }\end{array}$ & «percentual de \\
\hline
\end{tabular}

onde:

- "leitos ocupados" é o correspondente ao percentual de leitos ocupados, em relação ao total de leitos existentes, preconizado ou pelo autor ou encontrado no Hospital Universitário;

- "horas de assistência de enfermagem" é igual ao número de horas/enfermagem dispendidas com o cuidado ao paciente nas 24 horas, e varia por autor ou tipo de cuidado para adulto e pediatria;

- "dias da semana," em número de sete;

- "jornada de trabalho", isto é, o número de horas semanais trabalhadas $(40 \mathrm{~h})$;

- "percentual de absenteísmo" corresponde à cobertura das ausências ocasionadas por licenças, faltas, férias e folgas. Também varia por autor e pelo encontrado no Hospital Universitário. 
Para registro dos dados necessários à determinação do tipo de cuidado que o paciente requer, foi elaborado um mapa (ANEXO I), com identificação da clínica, contendo trinta e uma caselas correspondentes ao número de dias da coleta, para serem preenchidas com o número de leitos ocupados, em função do tipo de cuidados. Estes pacientes foram classificados segundo as definições estabelecidas por ALCALA: (Anexo II).

As enfermeiras que participaram do plano piloto foram as mesmas que realizaram a coleta de dados. O ensaio preliminar foi executado durante os 15 dias que antecederam a realização do estudo nas mesmas unidades de internação, a fim de permitir a avaliação do instrumento, o preparo das enfermeiras na determinação do tipo de cuidados e a demonstração da viabilidade do mesmo.

A coleta de dados foi realizada durante a visita diária aos pacientes, às 8 horas, por três enfermeiras da Clínica Médica e duas da Clínica Pediátrica. Nesta visita, que é de rotina no HU, a enfermeira faz, com cada paciente, uma entrevista, exame físico sumário, avalia a evolução do caso e faz a prescrição de enfermagem.

Durante a entrevista e exame físico, na fase de realização do estudo, eram identificadas: condiçōes de movimentação e deambulação, tipo de isolamento, presença de sondas, drenos, catéteres, venoclises, curativos, lesões, orientação auto e alo psiquica e condições emocionais dos pacientes. Na Pediatria foi ainda levado em consideração o tipo de berço (isolete, berço aquecido ou berço comum). Na avaliação e prescriação de enfermagem era feita comparação com a evolução dos dias anteriores, e verificação do aparecimento e resolução de novos problemas, assim como do número de cuidados a serem prestados. Cada paciente foi visitado tantas vezes quantos os dias em que permaneceu hospitalizado neste mês de estudo.

Para cálculo do percentual de absenteísmo, em ambas as clínicas, foi utilizada a fórmula encontrada em MEZOMO $^{6}$ onde:

«homens/dia perdido» $\times 100$

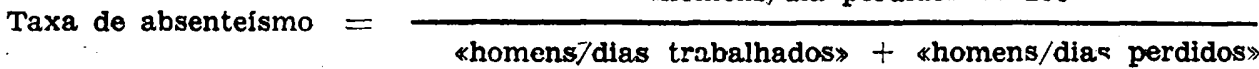

Para homem/dia perdido foram consideradas folgas, férias, faltas, licenças e vagas por demissões, e para homem/dia trabalhado, os 31 dias trabalhados de cada funcionário no mês de maio.

$O$ resultado foi, para a Pediatria, de $33 \%$ de absenteísmo e para a Clínica Médica, de $37 \%$.

\section{RESULTADOS}

A fim de facilitar a compreensão dos resultados, optou-se pela apresentação, inicialmente, dos cálculos elaborados, utilizando-se os índices, por autor, sendo: 1. ABBOUD ${ }^{1}, 2 . \operatorname{ALCALA}^{2}$ e 3 . os encontrados no 
HU, para a Clínica Pediátrica e Clínica Médica, seguidos das tabelas com distribuição funcional.

\section{CLINICA PEDIATRICA}

1. 35 leitos; $80 \%$ de ocupação; $20 \%$ absenteísmo.

$$
\frac{28 \times 4,9 \times 7}{40}+20 \%=28,85
$$

Pelos índices recomendados por $\mathrm{ABBOUD}^{1}$, obteve-se um total de 29 funcionários.

2. 35 leitos; $80 \%$ de ocupação; $30 \%$ absenteísmo, com $60 \%$ dos leitos - ocupados para cuidados mínimos, $30 \%$ para cuidados intermediários e $10 \%$ para cuidados intensivos.

a. Cuidados Mínimos

$$
=\frac{16,8 \times 4,5 \times 7}{40}+30 \%=17,20
$$

b. Cuidados Intermediários

$$
=\frac{8,4 \times 5,5 \times 7}{40}+30 \%=10,50
$$

c. Cuidados Semi-Intensivos

$$
=\frac{2,8 \times 10 \times 7}{40}+30 \%=6,37
$$

Realizado o cálculo, de acordo com os indices estabelecidos por ALCALA ${ }^{2}$, obteve-se um total de 34,07 funcionários.

3. 35 leitos; $91,42 \%$ de ocupação e $33 \%$ de absenteísmo.

Utilizou-se a classificação de ALCALA 2, mas com a distribuição de ocupação em 6,25\% para cuidados minimos; $15,62 \%$ para cuidados intermediários; $65,62 \%$ para cuidados semi-intensivos e $12,5 \%$ para cuidados intensivos, resultantes dos dados colhidos no mês em estudo.

a. Cuidados Mínimos

$$
=\frac{2 \times 4,5 \times 7}{40}+33 \%=2,09
$$

b. Cuidados Intermediários

$$
=\frac{5 \times 5,5 \times 7}{40}+33 \%=6,40
$$


c. Cuidados Semi-Intensivos

$$
=\frac{21 \times 10 \times 7}{40}+33 \%=48,87
$$

d. Cuidados Intensivos

$$
=\frac{4 \times 18 \times 7}{40}+33 \%=16,75
$$

Realizado o cálculo, de acordo com os indices encontrados no $\mathrm{HU}$, obteve-se um total de 74,11 funcionários.

\section{CLINICA MÉDICA}

1. 35 leitos; $80 \%$ de ocupação e $20 \%$ de absenteísmo.

$$
=\frac{28 \times 3,4 \times 7}{40}+20 \%_{c}^{\prime}=19,99
$$

Pelos indices recomendados por $\mathrm{ABBOUD}^{1}$, obteve-se um total de 20 funcionários.

2. 35 leitos; $80 \%$ de ocupação; $30 \%$ de absenteísmo e com $30 \%$ dos leitos ocupados para cuidados mínimos; $60 \%$ para cuidados intermediários e $10 \%$ para cuidados semi-intensivos.

a. Cuidados Mínimos

$$
=\frac{8,4 \times 2,5 \times 7}{40}+30 \%=4,76
$$

b. Cuidados Intermediários

$$
=\frac{16,8 \times 4,5 \times 7}{40}+30 \%=17,29
$$

c. Cuidados Semi-Intensivos

$$
=\frac{2,8 \times 10,0 \times 7}{40}+30 \%=6,37
$$

Realizado o cálculo, de acordo com os indices estabelecidos por ALCALA ${ }^{2}$, obteve-se um total de 28,42 funcionários.

3. 35 leitos; $91,42 \%$ de ocupação e $37 \%$ de absenteísmo. Utilizou-se a classificação de ALCALA ${ }^{2}$ mas com a distribuição de ocupação em $53,12 \%$ de cuidados mínimos; $34,37 \%$ de cuidados intermediários; $9,37 \%$ de cuidados semi-intensivos e $3,12 \%$ de cuidados intensivos, que foi a realidade no mês em estudo. 
a. Cuidados Mínimos

$$
=\frac{17 \times 2,5 \times 7}{40}+37 \%=10,18
$$

b. Cuidados Intermediários

$$
=\frac{11 \times 4,5 \times 7}{40}+37 \%=11,86
$$

c. Cuidados Semi-Intensivos

$$
=\frac{3 \times 10 \times 7}{40}+37 \%=7,19
$$

d. Cuidados Intensivos

$$
=\frac{1 \times 18 \times 7}{40}+37 \%=4,31
$$

Realizado o cálculo, de acordo com os índices encontrados no HU, obteve-se um total de 33,54 funcionários.

T A B E L A 1

DISTRIBUIÇAO DO PESSOAL DE ENFERMAGEM, POR CATEGORIA

\begin{tabular}{|c|c|c|c|c|c|c|}
\hline \multirow[b]{3}{*}{$\begin{array}{l}\text { Categoria } \\
\text { Funcional }\end{array}$} & \multicolumn{6}{|c|}{ Distribuigão do Pessoal } \\
\hline & \multicolumn{2}{|c|}{ Abboud } & \multicolumn{2}{|c|}{ Alcala } & \multicolumn{2}{|c|}{$\begin{array}{l}\text { Dept". de Assistência } \\
\text { de Enfermagem - HU }\end{array}$} \\
\hline & 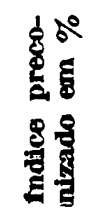 & $\frac{8}{5}$ & 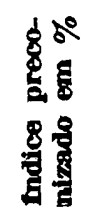 & $\frac{3}{3}$ & $\begin{array}{r}50 \\
8 \\
8 \\
8 \\
8 \\
8 \\
8\end{array}$ & 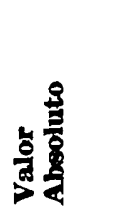 \\
\hline Enfermeira/o & 5 & 2 & 15 & 5 & 23 & 17 \\
\hline $\begin{array}{l}\text { Técnico de } \\
\text { Enfermagem }\end{array}$ & 10 & 3 & - & - & 3,5 & 3 \\
\hline $\begin{array}{l}\text { Auxiliar de } \\
\text { Enfermagem }\end{array}$ & 25 & 7 & 45 & 15 & 23,5 & 17 \\
\hline $\begin{array}{l}\text { Atendente de } \\
\text { Enfermagem }\end{array}$ & 60 & 17 & 40 & 14 & 50 & 37 \\
\hline Total & 100,0 & 29 & 100,0 & 34 & 100,0 & $\mathbf{7 4}$ \\
\hline
\end{tabular}

FUNCIONAL SEGUNDO ABBOUD1, ALCALA2 E DADOS

DO DEPARTAMENTO DE ASSISTENCIA DE ENFERMAGEM

DO HU, PARA A CLINICA PEDIATRICA, 1986 
T A B EL A 2

DISTRIBUTÇAO DO PESSOAL DE ENFERMAGEM, POR CATEGORIA FUNCIONAL SEGUNDO ABBOUD1, ALCALA2 E DADOS DO DEPARTAMENTO DE ASSISTENCIA DE ENFERMAGEM DO HU, PARA A CLINICA MEDICA, 1986

\begin{tabular}{|c|c|c|c|c|c|c|}
\hline \multirow[b]{3}{*}{$\begin{array}{l}\text { Catogoria } \\
\text { Funcional }\end{array}$} & \multicolumn{6}{|c|}{ Distribuigão do Pessoal } \\
\hline & \multicolumn{2}{|c|}{ Abboud } & \multicolumn{2}{|c|}{ Alcala } & \multicolumn{2}{|c|}{$\begin{array}{l}\text { Dept. de Assistência } \\
\text { de Enfermagem - HU }\end{array}$} \\
\hline & 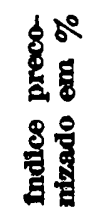 & 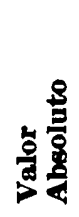 & 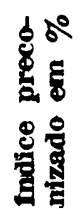 & 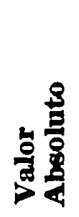 & 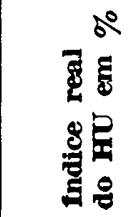 & 岁总 \\
\hline Enfermeira/o & 5 & 1 & 15 & 4 & 23 & 8 \\
\hline $\begin{array}{l}\text { Técnico de } \\
\text { Enfermagem }\end{array}$ & 10 & 2 & - & - & 3,5 & 1 \\
\hline $\begin{array}{l}\text { Auxiliar de } \\
\text { Entermagem }\end{array}$ & 25 & 5 & 45 & 13 & 23,5 & 8 \\
\hline $\begin{array}{l}\text { Atendente de } \\
\text { Enfermagem }\end{array}$ & 60 & 12 & 40 & 11 & 50 & 17 \\
\hline Total & 100,0 & 20 & 100,0 & 28 & 100,0 & 34 \\
\hline
\end{tabular}

\section{DISCUSSĀO}

No presente estudo de cálculo de pessoal realizado no Hospital Universitário por enfermeiras do Departamento de Assistência de Enfermagem, os resultados apresentados evidenciam uma variação entre os indices dos autores citados e os dados reais do $\mathrm{HU}$.

O emprego de um determinado critério pode ser um ponto de partida para se efetuar estudo desta natureza, entretanto, as diferenças encontradas precisam ser analisadas à luz das características do local onde se realizou a pesquisa; isto porque existem fatores que influenciam sobremaneira o comportamento dos índices; portanto, o emprego de um critério definido pode ser um ponto de partida para se calcular o pessoal para o serviço de enfermagem. Entretanto, a diferença do número de funcionários da Clínica Pediátrica, apresentado nos resultados, 29 para $\mathrm{ABBOUD}^{1}$ e 74 para o $\mathrm{HU}$, deve ser vista através dos índices utilizados. Esta variação aconteceu não só pela situação real do nível de assistência percentual de leitos ocupados, $91,42 \%$ contra $80 \%$ preconizados pelos dois autores; $33 \%$ de absenteísmo encontrados no HU contra $20 \%$ referidos por $\mathrm{ABBOUD}^{1}$ e $30 \%$ por ALCALA $^{2}$, mas principalmente pela classificação do tipo de cuidado. Comparando os resultados da Clínica Pediátrica com os da Clínica Médica, onde é menor a diferença entre os dados verifica-se que, embora o índice de ocupação de leitos tenha sido mantido em $91,42 \%$ e o absenteísmo no HU tenha sido maior (37\%), prin- 
cipalmente em relação a ABBOUD ${ }^{1}$, a influência da classificação por tipo de cuidado torna-se evidente. A partir destes achados, acredita-se que, para a determinação dos índices compativeis com a realidade, seja necessário manter-se uma classificação diária dos pacientes, por tipo de cuidados, e sugere-se, pelo menos, 06 meses de estudo, para que, de fato, este índice seja significativo. Isto porque podem ocorrer variações quanto à gravidade dos pacientes, com modificação dos percentuais do tipo de cuidado.

Considera-se importante que a enfermeira, que procede à classificação dos pacientes, obedeça a um padrão fixado para realizar este procedimento com objetividade e para diminuir a possibilidade de impressões subjetivas. $\overline{\mathrm{E}}$ recomendável que, de início, o trabalho seja feito em dupla, até que se obtenha maior segurança.

Quanto ao indice de absenteísmo encontrado neste estudo, pode-se inferir que o fato de ser mais alto que o apontado pelos autores consultados, seja decorrente de ser o HU um hospital escola, com um quadro de funcionários de enfermagem predominantemente feminino.

Com relação aos índices de distribuição por categoria funcionał, Tabelas 1 e 2, observa-se que ABBOUD ${ }^{1}$ e ALCALA ${ }^{2}$ estabeleceram percentuais menores para Enfermeira do que os encontrados no HU, enenquanto que, neste, o percentual das outras categorias é inferior ao dos autores citados, o que pode minimizar os custos. Discorrer sobre esta diferença implicaria em questionar diretamente a qualidade da assistência.

A presença da enfermeira se fez necessária desde o treinamento de pessoal recém-admitido até o controlę da qualidade de assistência prestada pelo serviço, inclusive na manutenção de um sistema de educação continuada, prestação de assistência seletiva e prescrição de enfermagem. Estas tarefas não podem ser delegadas aos outros elementos da equipe e demandam não só preparo profissional inicial como aperfeiçoamento contínuo do corpo de enfermeiras que respondem pela Instituição.

\section{CONCLUSĀO}

O número total de funcionários necessários à prestação da assistência de enfermagem em Clínica Pediátrica, segundo ABBOUD $^{1}$ foi 29; ALCALA 2, 34; Departamento de Enfermagem do HU, 74. Para a Clínica Médica, segundo ABBOUD ${ }^{1}$ foi 20; ALCALA ${ }^{2}$, 28; Departamento de Assistência de Enfermagem do HU, 34.

Existe acentuada diferença nos resultados obtidos com a utilização de diferentes índices para a Clínica Pediátrica. O mesmo não ocorreu em Clínica Médica, embora se evidencie razoável diferença.

A classificação dos pacientes da unidade ou instituição, por tipo de cuidado, torna o cálculo de pessoal mais próximo da necessidade real. Para tanto é necessário estabelecer índices que sejam compativeis com a realidade da Instituição. 
CAMPEDELLI, M.C.; TAKITO, C.; SANCINETTI, T.R.; BENKO, M.A. Nursing personnel budgeting: a nurse's function. Rev. Esc. Enf. USP, Säo Paulo, 21 (1): 3-15, Apr. 1987.

The authors analyse the problem faced by the Nursing Service of a hospital when budgeting nursing personnel for patient care. They refer that the hospitals, at first, may use prestablished calculation in the literature, but they emphasize that later on, the estimatis must be corrected by their observation of the daily number of hours of care required by the patient in that particular situation and other local factors which interfere with nursing assistance. hospital.

UNITERMS: Nursing staff, hospital. Nursing administration. Nursing service,

\section{REFERENCIAS BIBLIOGRAFICAS}

1. ABBOUD, M. Dotaç̃o de pessoal na organizacão hospitalar. São Paulo, Escola de Administração de Empresas de São Paulo da FGV/HC/FMUSP, s.d. 9p. (apostila).

2. ALCALA, M.U. et alli. Cálculo de pessoal: estudo preliminar para estabelecimento de quadro de pessoal de enfermagem na superintendência médico hospitalar de urgência. São Paulo, Superintendência: Médico Hospitalar de Urgência, 1982 . 47p.

3. ATALA, A. Cálculo de pessoal para os serviços paramédicos de um hospital geral de 300 leitos. Rev. paul. Hosp., Săo Paulo, 26(6):244-56, jun. 1978.

4. DUTRA, V. de O. Administraçăo de recursos no hospltal. In: GONÇALVES, E.L. o hospital o a viš̃o administrativa contemporanea. Săo Paulo, Ploneira, 1983. cap. 2, p.67-114.

5. FELDMANN, M.A. Administraçio do servico de enfermagem. Săo Paulo, Uniăo social Camiliana, s.d. 138p.

6. MEZOMO, J.C. Absentelsmo. In:-—. Administraça dos recursos humanos no hospítal. São Paulo, Centro săo Camilo de Desenvolvimento em Administração da Saúde, 1981. p.167.

7. OGUISsO, T. et alii. O sistema empresarial na locação de pessoal de enfermagem para o INPS. Rev. Bras. Ent., Rio de Janeiro, 23(1/2):117-30, jan./jun. 1970.

Recebido para publicacăo em 4/11/86.

Aprovado para publicacão em 27/4/87. 


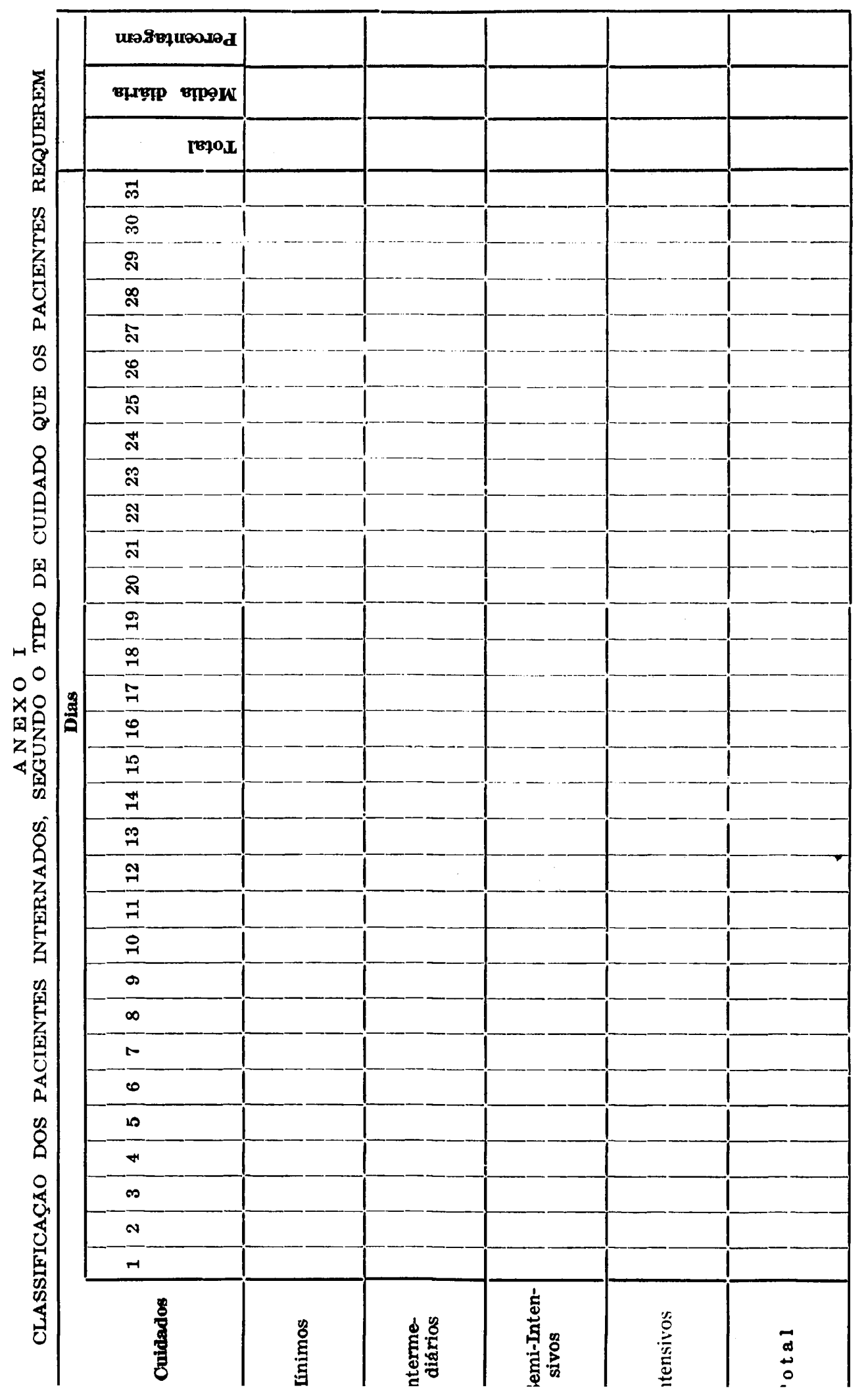




\section{CUIDADOS INTENSIVOS}

"Cuidados prestados a pacientes em estado crítico, que estão incapacitados de comunicar suas necessidades e que requerem cuidados e observaçðes de enfermagem concentrados. Estes pacientes necessitam de local e ambiente próprios a seu estado, munido de recursos materiais, equipamentos e drogas de emergência, bem como de profissionais altamente qualificados".

\section{CUIDADOS SEMI-INTENSIVOS}

"Cuidados prestados a pacientes que têm dependência total de enfermagem, para manter suas necessidades básicas, sem contudo apresentar risco de vida imediato; o cuidado e a observação de enfermagem não é tão concentrado como no paciente de cuidados intensivos, contudo exigem instalaçð̃es adequadas e pessoal profissional qualificado".

\section{CUIDADOS INTERMTEDIRIOS}

"Cuidados prestados a pacientes que exigem uma quantidade moderada de atenção de enfermagem, incluindo neste grupo os pacientes que estấo começando a participar do cuidado de si próprio".

\section{CUIDADO MTNIMO OU AUTO-CUIDADO}

"Cuidado de pacientes ambulantes, fisicamente auto-suficientes, que requerem serviços de diagnóstico terapêutico ou que passam estar em convalescência; as ações da enfermagem estão voltadas para possível controle terapéutico, curativos - principalmente para a orientação e instrução de medidas de promoçáo, recuperação e reabilitação. Aqui o paciente é instrufdo no auto-cuidado, dentro dos limites de sua enfermidade". 